\title{
Dressed states and spectra in quasicontinuum excitation
}

\author{
S. Tarzi \\ Optics Section, Blackett Laboratory, Imperial College of Science and Technology, London SW72BZ, United Kingdom \\ P. M. Radmore \\ Department of Electronic and Electrical Engineering, University College, University of London, \\ Torrington Place, London, WCIE 7JE, United Kingdom
}

(Received 3 December 1987)

\begin{abstract}
We consider a system in which a ground state is coupled to a general quasicontinuum of levels and to a true continuum. In the long-time limit, the true continuum spectrum can exhibit zeros, the positions of which depend on the energies of the quasicontinuum levels. The initial conditions determine the existence of these zeros. We describe this phenomenon by studying dressed states for the complete system and in particular derive dressed states which are ground-state independent. We use these to explain the locations of the zeros and their dependence on the initial conditions.
\end{abstract}

\section{INTRODUCTION}

The time evolution of a superposition of quasicontinuum levels excited from a single ground state has been extensively studied. ${ }^{1-3}$ Much of this work has discussed the model of Bixon and Jortner, ${ }^{2}$ consisting of the excitation of a single ground state to an equally spaced quasicontinuum (QC) of levels. The dynamics of the ground-state population exhibits a complicated time evolution beginning with a Weisskopf-Wigner decay; subsequent dephasing and partial reconstruction of the population occur. We have recently ${ }^{1}$ discussed a semiperturbative treatment for a general QC in which one QC level is resonant with the ground state and is treated nonperturbatively and the other QC levels act to perturb this twostate evolution.

In this paper the system we consider consists of a ground state coupled to a general QC of levels and to a true continuum. We examine the spectrum of final states in the true continuum. This spectrum can exhibit zeros, the positions of which are determined by the energies of the QC levels and the existence of which is determined by initial conditions. We obtain a general expression (for any $\mathrm{QC}$ ) of the true continuum spectrum and consider the special cases of first, a Bixon-Jortner QC, and second, a Rydberg series. We then study the dressed states ${ }^{4,5}$ for the whole system and derive "sparse" dressed states which contain few continuum levels and, more importantly, no contribution from the ground state. We show that the initial population in one of these sparse dressed states determines the existence of the corresponding zero in the spectrum. This is a similar mechanism to population trapping in certain systems ${ }^{6}$ where the population initially in a sparse dressed state is immune to photoionization. In Sec. II we derive a general expression for the spectrum. Section III discusses the special cases with particular reference to the initial conditions. The twophoton ionization problem discussed by $\mathrm{Knight}^{7}$ is also seen as a special case of our general treatment. In Sec. IV we discuss the total dressed states and explain the observed features using the sparse dressed states.

\section{BASIC EQUATIONS}

The system under consideration is that shown in Fig. 1, where a single discrete state $|0\rangle$ is coupled to both a true continuum of states $\{|f\rangle\}$ and a quasicontinuum of discrete states $\{|b\rangle\}$. For simplicity we take the matrix element between $|0\rangle$ and any state $|f\rangle$ to be $V_{0}$ (independent of $f$; ; that between $|0\rangle$ and state $|b\rangle$ is denoted $V_{b}$. A special case is the extended Bixon-Jortner model which we have studied recently. ${ }^{1}$ The equations of motion for the probability amplitudes $c_{0}(t), c_{f}(t)$, and $c_{b}(t)$ corresponding to states $|0\rangle,|f\rangle$, and $|b\rangle$ are

$$
\begin{aligned}
& \dot{c}_{0}=-i \sum_{b} V_{b} c_{b} e^{-i \Delta_{0 b^{t}}}-i \int_{f} V_{0} c_{f} e^{-i \Delta_{0 f^{t}}}, \\
& \dot{c}_{b}=-i V_{b} c_{0} e^{i \Delta_{0 b^{t}}}, \\
& \dot{c}_{f}=-i V_{0} c_{0} e^{i \Delta_{0 f^{t}},}
\end{aligned}
$$

where $\Delta_{0 b}$ and $\Delta_{0 f}$ are the detunings between $|0\rangle$ and $|b\rangle$, and between $|0\rangle$ and $|f\rangle$, respectively. We now put

$$
c_{b} e^{-i \Delta_{0 b} t}=a_{b}, \quad c_{f} e^{-i \Delta_{0 f} t}=a_{f}
$$

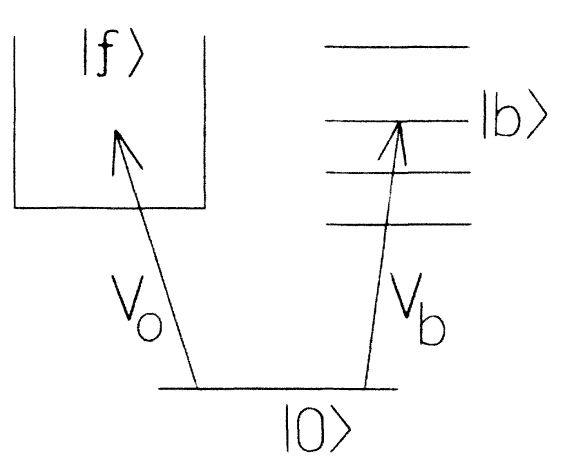

FIG. 1. Level scheme with the ground state coupled to an arbitrary QC and to a true continuum. 
so that $(1)-(3)$ become

$$
\begin{aligned}
& \dot{c}_{0}=-i \sum_{b} V_{b} a_{b}-i V_{0} \int_{f} a_{f}, \\
& \dot{a}_{b}=-i \Delta_{0 b} a_{b}-i V_{b} c_{0}, \\
& \dot{a}_{f}=-i \Delta_{0 f} a_{f}-i V_{0} c_{0} .
\end{aligned}
$$

To solve these equations we Laplace transform (with variable $s$ ) and take general initial conditions for the discrete states:

$$
c_{0}(0)=A, \quad a_{b}(0)=B_{b}, \quad a_{f}(0)=0 .
$$

Equations (5) $-(7)$ become

$$
\begin{aligned}
& s \widetilde{c}_{0}-A=-i \sum_{b} V_{b} \widetilde{a}_{b}-i V_{0} \int_{f} \widetilde{a}_{f}, \\
& \left(s+i \Delta_{0 b}\right) \widetilde{a}_{b}-B_{b}=-i V_{b} \widetilde{c}_{0}, \\
& \left(s+i \Delta_{0 f}\right) \widetilde{a}_{f}=-i V_{0} \widetilde{c}_{0},
\end{aligned}
$$

where a tilde denotes the Laplace transform. Using the final-value theorem in (11) gives

$$
a_{f}(\infty)=-i V_{0} \widetilde{c}_{0}\left(s=-i \Delta_{0 f}\right)
$$

so that we require $\widetilde{c}_{0}$ to find the final-state spectrum. Substituting for $\widetilde{a}_{b}$ and $\widetilde{a}_{f}$ in (9) and using (10) and (11) gives

$$
\begin{aligned}
s \widetilde{c}_{0}-A= & -i \sum_{b} V_{b}\left(B_{b}-i V_{b} \widetilde{c}_{0}\right) /\left(s+i \Delta_{0 b}\right) \\
& -V_{0}^{2} \widetilde{c}_{0} \int_{f}\left(s+i \Delta_{0 f}\right)^{-1} .
\end{aligned}
$$

Assuming the states $\{|f\rangle\}$ form a true infinite continuum of density $1 / \delta$, then

$$
\int_{f}\left(s+i \Delta_{0 f}\right)^{-1}=\pi / \delta \text {. }
$$

Using (14) and rearranging in (13) to find $\widetilde{c}_{0}$ we have

$$
\widetilde{c}_{0}(s)=\frac{A-i \sum_{b} V_{b} B_{b} /\left(s+i \Delta_{0 b}\right)}{s+\gamma+\sum_{b} V_{b}^{2} /\left(s+i \Delta_{0 b}\right)},
$$

where $\gamma=\pi V_{0}^{2} / \delta$. From (15) the final-value theorem result (12) can be written

$$
a_{f}(\infty)=-i V_{0} \frac{A+\sum_{b} V_{b} B_{b} /\left(\Delta_{0 f}-\Delta_{0 b}\right)}{-i \Delta_{0 f}+\gamma+i \sum_{b} V_{b}^{2} /\left(\Delta_{0 f}-\Delta_{0 b}\right)}
$$

so that the spectrum of true continuum final states is

$$
\left|a_{f}(\infty)\right|^{2}=V_{0}^{2} \frac{\left[A+\sum_{b} V_{b} B_{b} /\left(\Delta_{0 f}-\Delta_{0 b}\right)\right]^{2}}{\gamma^{2}+\left[\Delta_{0 f}-\sum_{b} V_{b}^{2} /\left(\Delta_{0 f}-\Delta_{0 b}\right)\right]^{2}} .
$$

In Sec. III we shall examine particular quasicontinua and their associated spectra using the general result above.

\section{SPECIFIC QUASICONTINUA}

\section{A. The Bixon-Jortner quasicontinuum}

In this case we assume that one of the levels $|b\rangle$ is resonantly coupled to $|0\rangle$ and that $\Delta_{0 b}=b \Delta$, where $b$ is an integer, ${ }^{2}-\infty<b<\infty$, and $V_{b}=V_{1}$ (constant). Then

$$
\sum_{b}\left(\Delta_{0 f}-\Delta_{0 b}\right)^{-1}=\frac{\pi}{\Delta} \cot \left(\frac{\pi \Delta_{0 f}}{\Delta}\right),
$$

which is required in (17). We shall study two different sets of initial conditions.

(i) $c_{0}(0)=1, c_{b}(0)=0$ (so that $A=1, B_{b}=0$ ). From (17) we have

$$
\left|a_{f}(\infty)\right|^{2}=\frac{V_{0}^{2}}{\gamma^{2}+\left[\Delta_{0 f}-\frac{\pi V_{1}^{2}}{\Delta} \cot \left(\frac{\pi \Delta_{0 f}}{\Delta}\right)\right]^{2}}
$$

and for convenience put

$F=\pi^{2} V_{0}^{2} / \Delta^{2}, \quad G=\pi^{2} \gamma^{2} / \Delta^{2}, \quad H=\pi^{2} V_{1}^{2} / \Delta^{2}$

and $x=\pi \Delta_{0 f} / \Delta$. The resulting spectrum is

$$
S_{1}(x)=\frac{F \sin ^{2} x}{(x \sin x-H \cos x)^{2}+G \sin ^{2} x} .
$$

In Fig. 2 we plot this spectrum as a function of $x$ for $V_{0}=V_{1}=\gamma=\Delta=1$. The spectrum has zeros corresponding to the positions of the energies of the Bixon-Jortner levels. This may be thought of as a "multiple hole" in the same manner as that studied by $\mathrm{Knight}^{7}$ in two-photon ionization. His results can, of course, be retrieved by replacing the QC by a single level. The origin of these zeros (or holes) can be described by reference to dressed states for the whole system which we discuss in Sec. IV. In Fig. 3 we plot $S_{1}(x)$ from (21) as a function of $x$ for

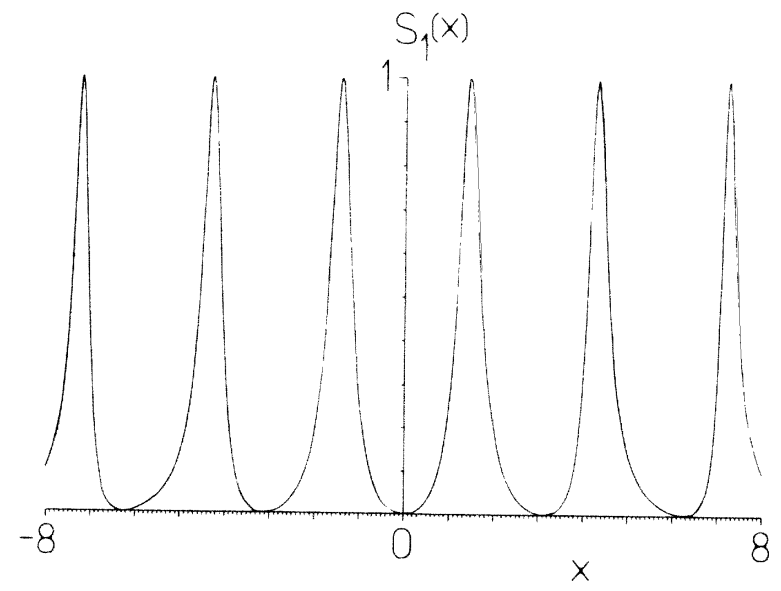

FIG. 2. Spectrum $S_{1}(x)$ from Eq. (21) for $V_{0}=V_{1}=\gamma=\Delta=1$ (Bixon-Jortner QC). 


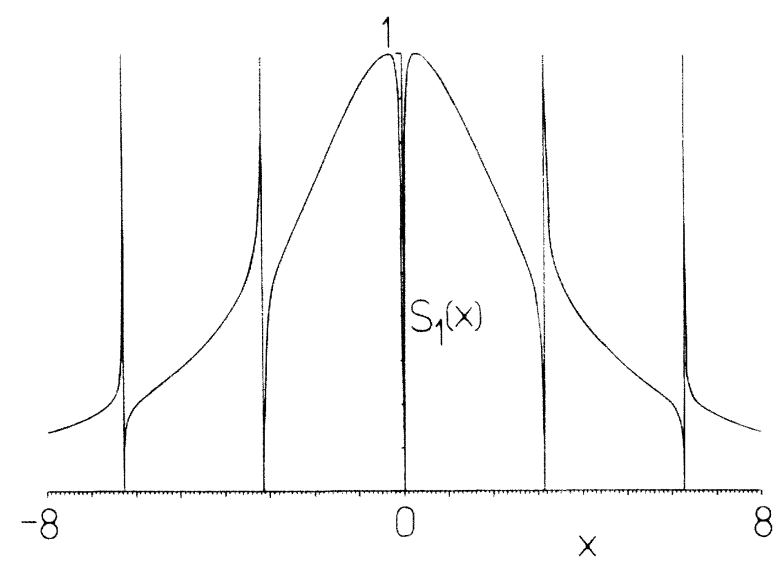

FIG. 3. Spectrum $S_{1}(x)$ from Eq. (21) for $V_{0}=\gamma=\Delta=1$, $V_{1}=0.1$ (Bixon-Jortner QC).
$V_{0}=\gamma=\Delta=1$ as before but with $V_{1}=0.1$, so that the Bixon-Jortner levels nonresonant with $|0\rangle$ are only weakly coupled. The spectrum has an underlying Lorentzian structure (a single state is strongly coupled to the true continuum) with a narrow hole at $x=0$, as would be expected from the calculations of Knight; ${ }^{7}$ in addition there are the zeros at the energy positions of the BixonJortner levels and the approach to multiple confluences. ${ }^{8}$ We note here that the peaks all have the same height for the spectrum $S_{1}(x)$ in (21) since they occur where $x \sin x=H \cos x$ and $S_{1}(x)$ then has value $F / G$ (equal to 1 in Figs. 2 and 3).

(ii) $c_{0}(0)=0, c_{b=N}(0)=1, c_{b \neq N}(0)=0$ (so that $A=0$, $\left.B_{N}=1, B_{b \neq N}=0\right)$. Here we begin with all the population in one of the Bixon-Jortner levels $b=N$, where $N=0, \pm 1, \pm 2, \ldots$. In this case

$$
\left|a_{f}(\infty)\right|^{2}=\frac{V_{0}^{2} V_{1}^{2}}{\left(\Delta_{0 f}-N \Delta\right)^{2}\left\{\gamma^{2}+\left[\Delta_{0 f}-\frac{\pi V_{1}^{2}}{\Delta} \cot \left[\frac{\pi \Delta_{0 f}}{\Delta}\right]\right]^{2}\right\}}
$$

so that using the same notation as before, in (20),

$S_{2}(x)=\frac{F H \sin ^{2} x}{(x-N \pi)^{2}\left[(x \sin x-H \cos x)^{2}+G \sin ^{2} x\right]}$.

Note that the existence of population initially in level $|b=N\rangle$ of the QC generates a factor $(x-N \pi)^{2}$ which will cancel the corresponding zero (of the factor $\sin ^{2} x$ at $x=N \pi$ ) at the position of this Bixon-Jortner level in the same manner as at a confluence. ${ }^{8}$ We shall describe this feature again in Sec. IV. In Fig. 4 we plot $S_{2}(x)$ against $x$ for $V_{0}=V_{1}=\gamma=\Delta=1$ and $N=0$. The zero at $x=0$ has been removed and the spectrum retains the pair of central peaks from Fig. 2, arising from the strongly coupled states $|0\rangle$ and $|b=0\rangle$. If we decrease the coupling $V_{1}$ or increase $\gamma$ we should see the two peaks merge into a single central peak. To illustrate the effect of large $\gamma$ we plot $S_{2}(x)$ against $x$ for $V_{0}=V_{1}=\Delta=1, N=0$, and $\gamma=4$

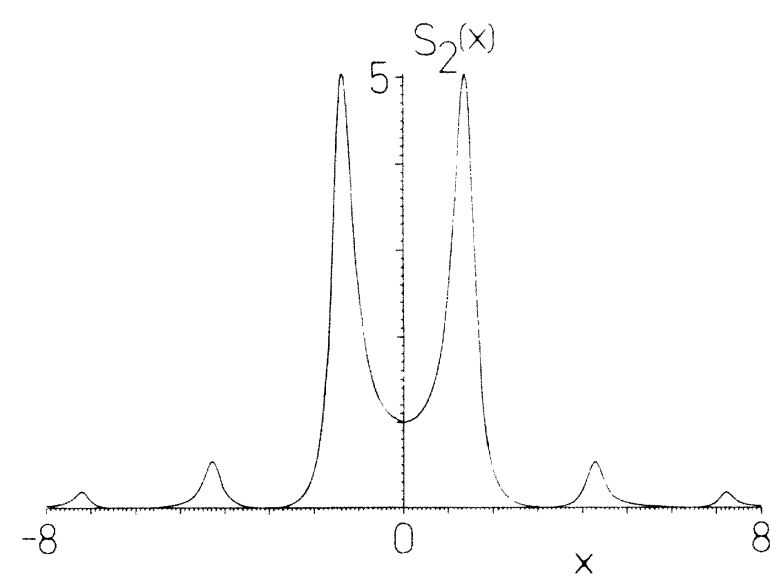

FIG. 4. Spectrum $S_{2}(x)$ from Eq. (23) for $V_{0}=V_{1}=\gamma=\Delta$ $=1, N=0$ (Bixon-Jortner QC). in Fig. 5. A single peak is observed but note that the other zeros persist. In Fig. 6 we again choose $V_{0}=V_{1}=\Delta=\gamma=1$, but put $N=1$ to show an asymmetric spectrum. Now the zero corresponding to $x=\pi$ at the energy position $|b=1\rangle$ has been removed.

\section{B. Rydberg series}

We take $\{|b\rangle\}$ to be the levels of a Rydberg series $|n\rangle$ with the level $|n=N\rangle$ resonantly coupled to the discrete state $|0\rangle$. The detunings are

$$
\Delta_{0 n}=D\left(1-\frac{N^{2}}{n^{2}}\right),
$$

and the initial conditions $c_{0}(0)=1, c_{n}(0)=0$ and $c_{f}(0)=0$ to begin with. Hence in (17), $A=1$ and $B_{b}=0$, and the spectrum is

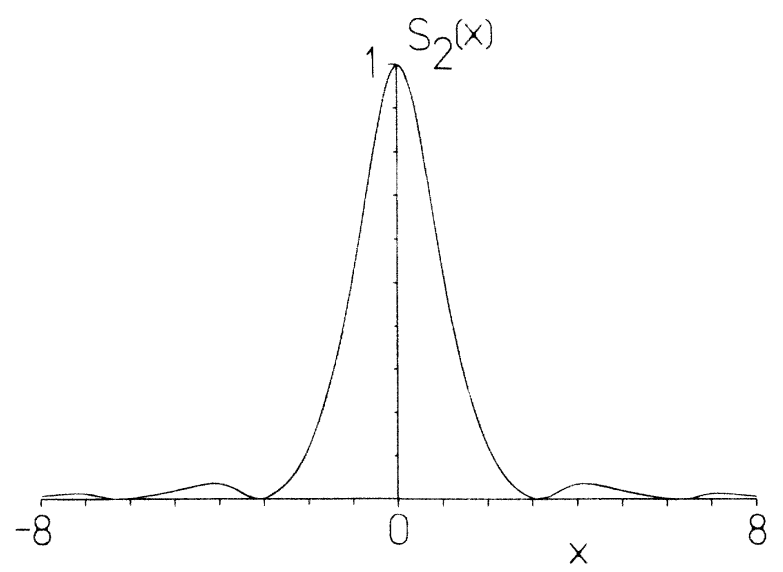

FIG. 5. Spectrum $S_{2}(x)$ from Eq. (23) for $V_{0}=V_{1}=\Delta=1$, $N=0$, and $\gamma=4$ (Bixon-Jortner QC). 


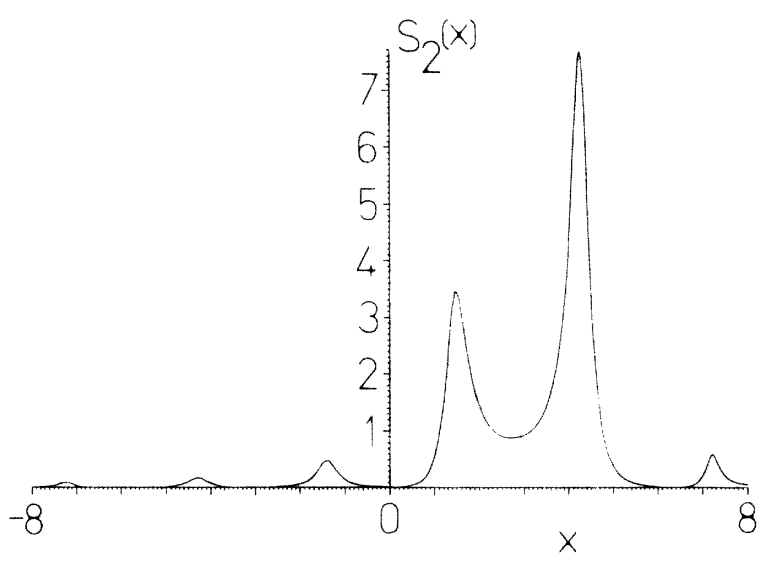

FIG. 6. Spectrum $S_{2}(x)$ from Eq. (23) for $V_{0}=V_{1}=\Delta=\gamma$ $=N=1$ (Bixon-Jortner QC).

$$
S_{1}(y)=\frac{P}{Q+[\pi y-\Theta(y)]^{2}},
$$

where

$P=\pi^{2} V_{0}^{2} / D^{2}, \quad Q=\pi^{2} \gamma^{2} / D^{2}, \quad y=\Delta_{0 f} / D$,

and

$$
\Theta(y)=\frac{\pi}{D^{2}} \sum_{n=1}^{\infty} \frac{V_{n}^{2}}{y-1+N^{2} / n^{2}} .
$$

Taking the matrix elements to be

$$
V_{n}^{2}=V^{2} / n^{3} \text {, }
$$

we may write (27) as

$$
\Theta(y)=R \sum_{n=1}^{\infty} \frac{1}{n\left[n^{2}(y-1)+N^{2}\right]},
$$

where

$$
R=\pi V^{2} / D^{2} .
$$

The function $\Theta(y)$ can be evaluated in closed form in terms of digamma functions. ${ }^{9}$ We plot $S_{1}(y)$ from (25) against $y$ in Fig. 7 for $P=Q=R=N=1$. Note that there is a zero in the spectrum at each position of a Rydberg level $\left(y=1-1 / n^{2}=0, \frac{3}{4}, \frac{8}{9}, \frac{15}{16}, \ldots\right)$. The zeros end at $y=1$, which corresponds to the upper limit of the Rydberg series and crowd together as $y$ tends to 1 from below as expected. ${ }^{10}$ In Fig. 8 we again plot $S_{1}(y)$ against $y$ with $P=Q=R=1$ but choose $N=2$ (and note that the zeros now occur at $y=1-4 / n^{2}=-3,0, \frac{5}{9}, \frac{3}{4}, \frac{21}{25}, \frac{8}{9}, \ldots$ ).

The zeros of $S_{1}(y)$ in (25) originate from the divergences in $\Theta(y)$ at the positions of the Rydberg levels. We can see from (17) that if initial conditions were chosen with all the population in one Rydberg level $N(A=0$, $B_{N}=1$ ), then the divergence in the denominator of (17) at that place is matched by that of the single term in the numerator. Hence the zero in the spectrum corresponding to the populated Rydberg level is removed and the $y$ dependence of the spectrum for $N=1$ is

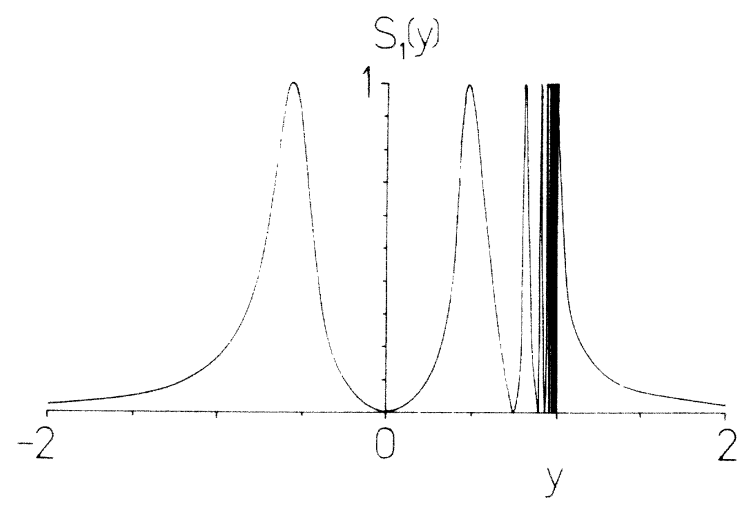

FIG. 7. Spectrum $S_{1}(y)$ from Eq. (25) for $P=Q=R=N=1$ (Rydberg QC).

$$
S_{2}(y) \propto y^{-2}\left\{Q+[\pi y-\Theta(y)]^{2}\right\}^{-1} .
$$

We illustrate this in Fig. 9 by plotting this spectrum normalized to unit height with $Q=R=N=1$. Note the absence of the zero at $y=0$ corresponding to the $n=1 \mathrm{Ryd}$ berg level.

It is also instructive to consider a Rydberg-like series where the detunings are as above, Eq. (24), but the matrix elements are taken to be

$$
V_{n}^{2}=V^{2} / n^{2}
$$

instead of (28). With this choice

$$
\Theta(y)=R \sum_{n=1}^{\infty} \frac{1}{n^{2}(y-1)+N^{2}} .
$$

This series can be summed analytically in terms of the coth function but we do not specifically need the expression here. With $\Theta(y)$ as in (33), we plot from (25) in Fig. 10 with $P=Q=R=N=1$ and compare this with Fig. 7. The two spectra are very similar: the zeros occur at the same positions in both. The particular form of the matrix elements does not affect these zeros but the peaks differ slightly in position and shape in the two figures. Again, a particular zero can be removed by initially populating the corresponding QC level. We will not show this here. The importance of initial conditions in determining the continuum population has been discussed, in a related context, by Agassi. ${ }^{11}$

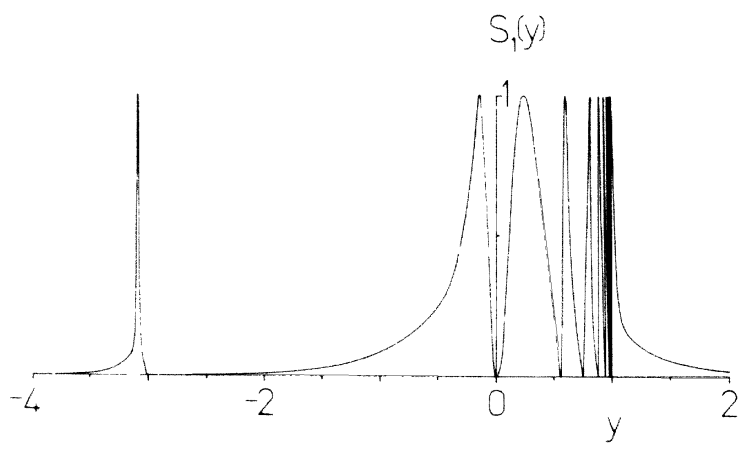

FIG. 8. Spectrum $S_{1}(y)$ from Eq. (25) for $P=Q=R=1$, $N=2$ (Rydberg QC). 


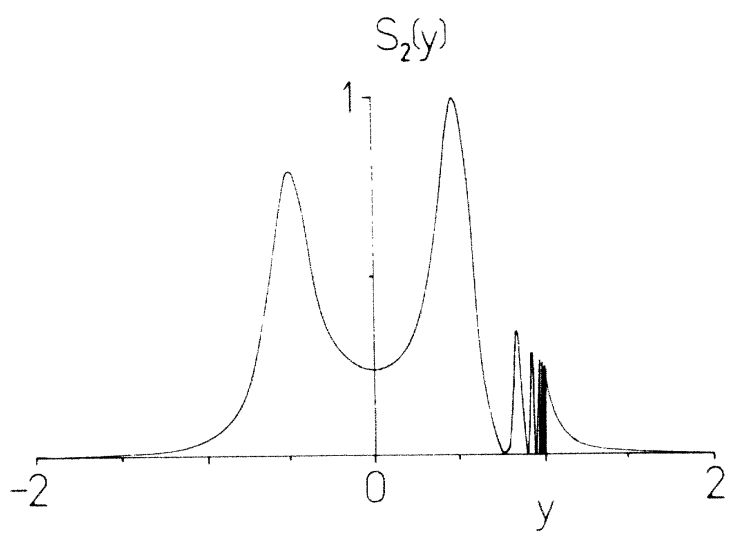

FIG. 9. Spectrum $S_{2}(y)$ from Eq. (31) for $Q=R=N=1$ normalized to unit height (Rydberg QC).

In Sec. IV we discuss the dressed states for the total system with a general QC and explain the above features in the spectra.

\section{DRESSED STATES}

We now discuss the dressed states for the whole system in Fig. 1 in order to more fully account for the features in the spectra of Sec. III. With labels 0 and 1 to denote the fields driving the $|0\rangle$ to $\{|f\rangle\}$ and $|0\rangle$ to $\{|b\rangle\}$ transitions, respectively, with photon numbers $n_{0}$ and $n_{1}$, we write

$$
\begin{aligned}
& |i\rangle=\left|0, n_{0}, n_{1}\right\rangle, \\
& |k\rangle=\left|f, n_{0}-1, n_{1}\right\rangle, \\
& |l\rangle=\left|b, n_{0}, n_{1}-1\right\rangle .
\end{aligned}
$$

The states have energies, say, $E_{i}, E_{k}$, and $E_{l}$. We wish to find eigenstates of the total Hamiltonian

$$
\hat{H}=\hat{H}_{0}+\sum_{l} \hat{V}_{i l}+\sum_{k} \hat{W}_{i k}
$$

where $\hat{H}_{0}|i\rangle=E_{i}|i\rangle$, and similarly for $|k\rangle$ and $|l\rangle$.

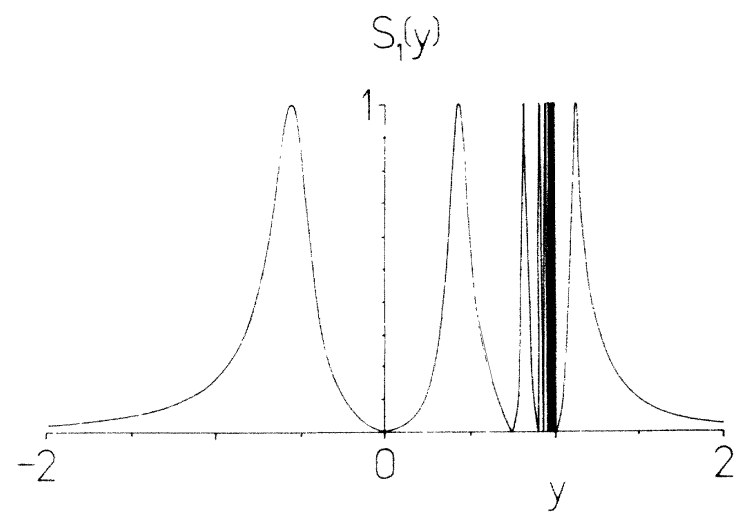

FIG. 10. Spectrum $S_{1}(y)$ from Eq. (25) with $\Theta(y)$ given by (33) and $P=Q=R=N=1$ (Rydberg-like QC). This is to be compared with Fig. 7.
The sums represent the possible couplings between $|i\rangle$ and any of the states $|l\rangle$ in the case of $V_{i l}$, and between $|i\rangle$ and any of the states $|k\rangle$ in the case of $\hat{W}_{i k}$. We require $|\varepsilon\rangle$, where $\hat{H}|\varepsilon\rangle=\varepsilon|\varepsilon\rangle$, and expand $|\varepsilon\rangle$ in terms of the basis states $(34)-(36)$ :

$$
|\varepsilon\rangle=P(\varepsilon)|i\rangle+\sum_{l} Q_{l}(\varepsilon)|l\rangle+\int d k c_{k}(\varepsilon)|k\rangle .
$$

The procedure is similar to that adopted by Fano and by Coleman et al.: ${ }^{5}$ Taking matrix elements of the eigenvalue equation for $|\varepsilon\rangle$ with each of $(34)-(36)$ in turn gives

$$
\begin{aligned}
& \left(E_{i}-\varepsilon\right) P(\varepsilon)+\sum_{l} Q_{l}(\varepsilon) V_{i l}+\int d k c_{k}(\varepsilon) W_{i k}=0, \\
& \left(E_{l}-\varepsilon\right) Q_{l}(\varepsilon)+P(\varepsilon) V_{i l}=0, \\
& \left(E_{k}-\varepsilon\right) c_{k}(\varepsilon)+P(\varepsilon) W_{i k}=0 .
\end{aligned}
$$

The sensitivity of the zeros in the spectra to initial conditions arises because of the existence of special dressed states, namely, states which contain no contribution from $|i\rangle$, so that if all atomic population begins in $|i\rangle$, then these dressed states are initially unpopulated. We first find these states. For them to exist there must be values of $\varepsilon$, $\bar{\varepsilon}$, say, such that $P(\bar{\varepsilon})=0$ [see (38)]. Hence, from (40) we have

$$
\left(E_{l}-\bar{\varepsilon}\right) Q_{l}(\bar{\varepsilon})=0 .
$$

Note that $(42)$ is a set of equations, one for each $l$. Now not all the $Q_{l}(\bar{\varepsilon})$ are zero (otherwise the dressed state contains no continuum states) so the only possibility is that $\bar{\varepsilon}$ equals one particular $E_{l}$ and that the corresponding $Q_{l}(\bar{\varepsilon})$ is the only nonzero one. We therefore have a set of possible solutions for $\bar{\varepsilon}$, namely, any of the energies of the QC levels (the positions where the zeros appear in the spectra). We conclude that there are as many of these sparse dressed states containing no contribution from $|i\rangle$ as there are QC levels and that their energies are those of the QC levels. To find these states we first use (40),

$$
Q_{l}(\varepsilon)=\frac{V_{i l} P(\varepsilon)}{\varepsilon-E_{l}} .
$$

From (41), since $E_{k}$ is a continuous variable, ${ }^{5}$

$c_{k}(\varepsilon)=P(\varepsilon) W_{i k}\left(\mathbf{P} \frac{1}{\varepsilon-E_{k}}+R(\varepsilon) \delta\left(\varepsilon-E_{k}\right)\right]$,

where $\mathrm{P}$ denotes the principal part and $R(\varepsilon)$ must be found self-consistently. Substituting (43) and (44) into (39) gives

$$
\begin{aligned}
& \left(E_{i}-\varepsilon\right) P(\varepsilon)+\sum_{l} \frac{V_{i l}^{2} P(\varepsilon)}{\varepsilon-E_{l}} \\
& \quad+\int d k P(\varepsilon) W_{i k}^{2}\left[\mathrm{P} \frac{1}{\varepsilon-E_{k}}+R(\varepsilon) \delta\left(\varepsilon-E_{k}\right)\right]=0 .
\end{aligned}
$$

This must be true for all $\varepsilon$, so that canceling $P(\varepsilon)$ gives 
$E_{i}-\varepsilon+\sum_{l} \frac{V_{i l}^{2}}{\varepsilon-E_{l}}+\mathbf{P} \int d k \frac{W_{i k}^{2}}{\varepsilon-E_{k}}+R(\varepsilon) W_{i \varepsilon}^{2}=0$

where $W_{i \varepsilon}$ means that value of $W_{i k}$ such that $E_{k}$ equals the chosen $\varepsilon$. Hence

$R(\varepsilon)=\frac{1}{W_{i \varepsilon}^{2}}\left(\varepsilon-E_{i}-\sum_{l^{\prime}} \frac{V_{i l^{\prime}}^{2}}{\varepsilon-E_{l^{\prime}}}-\mathbf{P} \int d k \frac{W_{i k}^{2}}{\varepsilon-E_{k}}\right)$.

From (44), we find that

$$
c_{k}\left(\varepsilon=E_{l}\right)=\lim _{\varepsilon \rightarrow E_{l}} W_{i k} P(\varepsilon) R(\varepsilon) \delta\left(\varepsilon-E_{k}\right) .
$$

Combining (47) and (48), we notice that as $\varepsilon \rightarrow E_{l}, P(\varepsilon)$ tends to zero whereas $R(\varepsilon)$ diverges. The only term which contributes, therefore, is

$$
\begin{aligned}
c_{k}\left(\varepsilon=E_{l}\right)= & W_{i k} \delta\left(E_{l}-E_{k}\right) \frac{1}{W_{i l}^{2}} \lim _{\varepsilon \rightarrow E_{l}} \\
& \times\left(-P(\varepsilon) \sum_{l^{\prime}} \frac{V_{i l^{\prime}}^{2}}{\varepsilon-E_{l^{\prime}}}\right),
\end{aligned}
$$

where $W_{i l}$ means $W_{i k}$ for that value of $k$ such that $E_{k}=E_{l}$. Again only one term in the sum in (49) will contribute,

$$
c_{k}\left(\varepsilon=E_{l}\right)=-\frac{W_{i k}}{W_{i l}^{2}} \delta\left(E_{l}-E_{k}\right) \lim _{\varepsilon \rightarrow E_{l}}\left(P(\varepsilon) \frac{V_{i l}^{2}}{\varepsilon-E_{l}}\right) .
$$

Substituting for $P(\varepsilon) /\left(\varepsilon-E_{l}\right)$ in (50) using (43), we find, finally,

$$
c_{k}\left(\varepsilon=E_{l}\right)=-\frac{W_{i k}}{W_{i l}^{2}} V_{i l} Q_{l}\left(\varepsilon=E_{l}\right) \delta\left(E_{l}-E_{k}\right) .
$$

The dressed state $\left|\varepsilon=E_{l}\right\rangle$ is, therefore, from (38),

$$
\begin{aligned}
\left|\varepsilon=E_{l}\right\rangle & =Q_{l}\left(E_{l}\right)|l\rangle+\int d k c_{k}\left(\varepsilon=E_{l}\right)|k\rangle \\
& =Q_{l}\left(E_{l}\right)\left(|l\rangle-\frac{V_{i l}}{W_{i l}}\left|s_{l}\right\rangle\right),
\end{aligned}
$$

where $\left|s_{l}\right\rangle$ means that continuum state $|k\rangle$ with energy equal to the chosen QC energy $E_{l}$. We therefore have a set of sparse dressed states, as many as there are QC states, each of which contains contributions only from a particular QC state and the true continuum state that is two-photon resonant with that QC state. This is a similar situation to that encountered in other systems with twophoton resonance, for example, three-level systems and other multilevel systems, ${ }^{6}$ where population trapping results. In the present system, if the population is initially in $|i\rangle$, then the sparse dressed states are unpopulated initially; zeros appear in the spectrum at places corresponding to the energies of these $|i\rangle$-independent states. If, however, we populate one of these states initially then the corresponding zero is removed. Hence we anticipate that the population in the true continuum states at the special positions should contain a nonvanishing term for long times that is proportional to the initial sparse dressed state population. We now show that this is the case.

The total wave function would be

$$
|\psi\rangle=\int A(\varepsilon) e^{-i \varepsilon t}|\varepsilon\rangle d \varepsilon
$$

so that

$$
\left\langle s_{l} \mid \psi\right\rangle=\int A(\varepsilon) e^{-i \varepsilon t} c_{s_{l}}(\varepsilon) d \varepsilon .
$$

Now

$$
c_{s_{l}}(\varepsilon)=P(\varepsilon) W_{i l}\left[\mathrm{P} \frac{1}{\varepsilon-E_{l}}+R(\varepsilon) \delta\left(\varepsilon-E_{l}\right)\right] .
$$

Using the expression for $R(\varepsilon),(47)$ in (55),

$$
\begin{aligned}
c_{s_{l}}(\varepsilon)= & P(\varepsilon) W_{i l} \mathbf{P} \frac{1}{\varepsilon-E_{l}} \\
& +P(\varepsilon) \frac{W_{i l}}{W_{i \varepsilon}^{2}} \delta\left(\varepsilon-E_{l}\right) \\
& \times\left(\varepsilon-E_{i}-\sum_{l^{\prime}} \frac{V_{i l^{\prime}}^{2}}{\varepsilon-E_{l^{\prime}}}-\mathbf{P} \int d k \frac{W_{i k}^{2}}{\varepsilon-E_{k}}\right),
\end{aligned}
$$

Substituting (56) into (54) we find

$$
\begin{aligned}
\left\langle s_{l} \mid \psi\right\rangle= & W_{i l} \mathbf{P} \int_{-\infty}^{\infty} \frac{P(\varepsilon) A(\varepsilon)}{\varepsilon-E_{l}} e^{-i \varepsilon t} d \varepsilon \\
& -\frac{1}{W_{i l}} A\left(E_{l}\right) e^{-i E_{l} t} V_{i l}^{2} \lim _{\varepsilon \rightarrow E_{l}} \frac{P(\varepsilon)}{\varepsilon-E_{l}} .
\end{aligned}
$$

Again using the expression for $P(\varepsilon) /\left(\varepsilon-E_{l}\right)$ from $(40)$ in (57) and taking the modulus squared, we have the population in the true continuum state $\left|s_{l}\right\rangle$,

$$
\begin{aligned}
\left|\left\langle s_{l} \mid \psi\right\rangle\right|^{2}=\mid & A\left(E_{l}\right) Q_{l}\left(E_{l}\right) \frac{V_{i l}}{W_{i l}} \\
& -\left.\frac{W_{i l}}{V_{i l}} e^{i E_{l} t} \int_{-\infty}^{\infty} Q_{l}(\varepsilon) A(\varepsilon) e^{-i \varepsilon t} d \varepsilon\right|^{2},
\end{aligned}
$$

If $|\psi(t=0)\rangle=|i\rangle$ then $A\left(E_{l}\right)=0$ and we know that $\left|\left\langle s_{l} \mid \psi\right\rangle\right|^{2} \rightarrow 0$ as $t \rightarrow \infty$. If $|\psi(t=0)\rangle=\left|l^{\prime}\right\rangle$ then $A\left(E_{l}\right) \neq 0$ and the first term on the right-hand side of (58) is nonzero. At long times the expression tends to a constant and the zero in the spectrum is removed.

\section{CONCLUSIONS}

We have shown how sparse dressed states containing contributions from a limited number of continuum levels are responsible for the appearance of zeros in our model QC problem. The existence of the zeros is determined by the initial populations in these sparse dressed states. We stress that the treatment is valid for a general QC so that the positions of the zeros follow the distribution of $\mathrm{QC}$ 
levels. In the Rydberg example, this results in a successively narrower gap between adjacent zeros as the Rydberg levels crowd together at the top of the series. The matrix elements in the case of the Rydberg-like series we considered do not, therefore, affect the positions of the zeros. These calculations indicate how clear the features of certain resonantly coupled multilevel problems may become when a dressed treatment is adopted.

\section{ACKNOWLEDGMENTS}

We would like to thank Dr. P. L. Knight and Dr. S. M. Barnett for useful discussions. One of us (S.T.) would like to thank the U.K. Science and Engineering Research Council for financial support.
${ }^{1}$ P. M. Radmore, S. Tarzi, and P. L. Knight, J. Mod. Opt. 34, 587 (1987).

${ }^{2}$ M. Bixon and J. Jortner, J. Chem. Phys. 48, 715 (1968).

${ }^{3}$ G. C. Stey and R. W. Gibberd, Physica (Utrecht) 60, 7 (1972); R. Lefebvre and J. Savolainen, J. Chem. Phys. 60, 2509 (1974); J. H. Eberly, J. J. Yeh, and C. M. Bowden, Chem. Phys. Lett. 86, 76 (1982); J. J. Yeh, C. M. Bowden, and J. H. Eberley, J. Chem. Phys. 76, 5936 (1982); P. W. Milonni, J. R. Ackerhalt, H. W. Galbraith, and M.-L. Shih, Phys. Rev. A 28, 32 (1983); E. Kyrölä, J. Opt. Soc. Am. B 1, 737 (1984); 3, 453 (1986); J. Phys. B 19, 1437 (1986); E. Kyrölä and J. H. Eberly, J. Chem. Phys. 82, 1841 (1985); A. A. Makarov, V. T. Platonenko, and V. V. Tyakht, Zh. Eksp. Teor. Fiz. 75, 2075 (1978) [Sov. Phys. - JETP 48, 1044 (1978)].

${ }^{4}$ D. Agassi and J. H. Eberly, Phys. Rev. A 34, 2843 (1986).
${ }^{5}$ U. Fano, Phys. Rev. 124, 1866 (1961); P. E. Coleman and P. L. Knight, J. Phys. B 15, L235 (1982); P. E. Coleman, P. L. Knight, and K. Burnett, Opt. Commun. 42, 171 (1982).

${ }^{6}$ P. M. Radmore and P. L. Knight, J. Phys. B 15, 561 (1982); P. M. Radmore, Phys. Rev. A 26, 2252 (1982).

${ }^{7}$ P. L. Knight, J. Phys. B 12, 3297 (1979).

${ }^{8}$ K. Rzążewski and J. H. Eberly, Phys. Rev. Lett. 47, 408 (1981); Phys. Rev. A 27, 2026 (1983); Z. Deng and J. H. Eberly, J. Opt. Soc. Am. B 1, 102 (1984).

${ }^{9}$ Handbook of Mathematical Functions, edited by $\mathbf{M}$. Abramowitz and I. A. Stegun (Dover, New York, 1964).

10J. P. Connerade, A. M. Lane, and M. A. Baig, J. Phys. B 18, 3507 (1985); J. P. Connerade and A. M. Lane, ibid. 20, 1757 (1987).

${ }^{11}$ D. Agassi, Phys. Rev. A 30, 2449 (1984). 